\title{
A scheme for tunable quantum phase gate and effective preparation of graph-state entanglement
}

\author{
Gong-Wei Lin ${ }^{1}, \mathrm{Xu}$-Bo Zou ${ }^{2}$, Ming-Yong $\mathrm{Ye}^{1}$, Xiu-Min Lin ${ }^{1}$ * and Guang-Can Guo ${ }^{2}$ \\ ${ }^{1}$ School of Physics and Optoelectronics Technology, \\ Fujian Normal University, Fuzhou 350007, People's Republic of China and \\ ${ }^{2}$ Key Laboratory of Quantum Information, Department of Physics, \\ University of Science and Technology of China, Hefei 230026
}

\begin{abstract}
A scheme is presented for realizing a quantum phase gate with three-level atoms, solid-state qubits - often called artificial atoms, or ions that share a quantum data bus such as a single mode field in cavity QED system or a collective vibrational state of trapped ions. In this scheme, the conditional phase shift is tunable and controllable via the total effective interaction time. Furthermore, we show that the method can be used for effective preparation of graph-state entanglement, which are important resources for quantum computation, quantum error correction, studies of multiparticle entanglement, fundamental tests of non-locality and decoherence.
\end{abstract}

\section{INTRODUCTION}

Since quantum computer and quantum communication can provide more powerful ability than the classical ones [1, 2], in the past few years, a variety of systems are explored for the realization of practical quantum information processors. Among them cavity quantum electrodynamics (QED) system [3, 4] and ion trap system [5, 6] are two of the ideal candidates for quantum computation and quantum communication. The distinctive feature of these two systems are (i) they allow the implementation of quantum gates between any set of (not necessarily neighboring) atoms, solid-state qubits - often called artificial atoms, or ions which share a single mode field in cavity QED systems or a collective vibrational state of trapped ions, (ii) the atoms or artificial atoms trapped in a high-Q cavity or the ions trapped in a potential trap have long decoherence times $[7,[8,[9,10,11]$. Because of these advantages many efforts have been devoted to the implementation of quantum logical gates and the generation of entangled states [12]. In particular, for cavity QED system several experiments have been reported about the generation of the EinsteinPodolsky-Rosen state [13] of two atoms, GreenbergerHorne-Zeilinger (GHZ) state 14] of three parties (two atoms plus one cavity mode), Schrödinger cat state [15], and Fock state [16] of a single-mode cavity field. Remarkably, in ion trap system maximally up to eight ions have been entangled [17].

In recent years, a special type of multipartite entangled states, so-called graph states [18, 19], have become the centre of attention. They can correspond to mathematical graphs, where each vertex represents a qubit prepared in the state $(|0\rangle+|1\rangle) / \sqrt{2}$ and each edge represents a two-qubit controlled-Z gate having been applied to the two connected qubits. An interesting feature is that many entanglement properties of graph states

*Electronic address: xmlin@fjnu.edu.cn are closely related to their underlying graphs [18, 19]. Besides their thought-provoking theoretical structure, graph states have also provided new insights into studies of nonlocality [20, 21] and become an interesting resource for multiparty quantum communication [22]. Special instances of graph states are codewords of various quantum error correcting codes [23], which are of central importance for protecting quantum states against decoherence in quantum computation [24, 25]. In particular, special instances of graph states have served as essential resources for quantum computation [26, 27]. Recently, much progress with preparation of arbitrary graph states has been made in the linear optics regime and the optical lattice [28, 29, 30]. In Ref. [31], experimental entanglement of six photons in graph states was reported.

In this paper, we propose an alternative scheme for quantum computation and quantum state engineering based on cavity QED or ion trap. The main result from this work is twofold: first, we propose a method for realizing a tunable quantum phase gate. The accumulated conditional phase shift $\phi$ can vary between 0 and $2 \pi$ by controlling the total effective interaction time. Compared with previous protocols for the tunable quantum phase gates [32, 33], our scheme encodes two logical states of a qubit on the two stable low energy states, and the conditional phase shifts are obtained without any real transitions of atomic internal states or cavity-photon or vibration-phonon population. In contrast to the scheme in Ref. [34], our method can be directly extended to construct multiple-qubit entangling gates. Second, a more important result here is that our method can be used for effective preparation of graph-state entanglement.

\section{THE FUNDAMENTAL MODEL AND TUNABLE QUANTUM PHASE GATE WITH THREE-LEVEL ATOMS}

Next we assume (without loss of generality) that $\mathrm{N}$ identical atoms, each having two low levels $|0\rangle,|1\rangle$ and a high level $|e\rangle$, simultaneously interact with a single-mode 
cavity and a classical field, as shown in Fig. 1. Both of

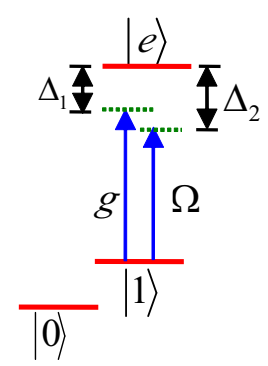

FIG. 1: (Color online) Configuration of the atomic level structure and relevant transitions. The states $|0\rangle$ and $|1\rangle$ correspond to two low levels while $|e\rangle$ is a high level. The transition $|1\rangle \rightarrow|e\rangle$ of each atom is driven by the cavity field and the classical pulse with the detunings $\Delta_{1}$ and $\Delta_{2}$, respectively. $g$ represents the coupling rate of the atom to cavity mode, and $\Omega$ is Rabi frequency of the classical pulse.

the cavity mode and the classical pulse are coupled to $|1\rangle \leftrightarrow|e\rangle$ transition of each atom. The Hamiltonian for the whole system in the interaction picture can be written as

$$
H_{\text {int }}=\sum_{j=1}^{N}\left(g_{j} e^{i \Delta_{1} t} a\left|e_{j}\right\rangle\left\langle 1_{j}\left|+\Omega_{j} e^{i \Delta_{2} t}\right| e_{j}\right\rangle\left\langle 1_{j}\right|\right)+H . c .,
$$

where $a$ is the annihilation operator of the cavity mode, $\Delta_{1}$ and $\Delta_{2}$ denote the detunings of the cavity mode and classical pulse from respective atomic transitions, $g_{j}$ represents the coupling rate of the jth atom to cavity mode, and $\Omega_{j}$ is Rabi frequency of the classical pulse for the jth atom. In the case of $\Delta_{1} \gg\left|g_{j}\right|$ and $\Delta_{2} \gg\left|\Omega_{j}\right|$, the high level $|e\rangle_{j}$ can be adiabatically eliminated, leading to

$$
\begin{aligned}
H_{e f f} & =\sum_{j=1}^{N}\left[-\frac{\left|g_{j}\right|^{2}}{\Delta_{1}} a^{\dagger} a\left|1_{j}\right\rangle\left\langle 1_{j}\left|-\frac{\left|\Omega_{j}\right|^{2}}{\Delta_{2}}\right| 1_{j}\right\rangle\left\langle 1_{j}\right|\right. \\
& \left.-\left(\lambda_{j} a e^{-i \delta t}+\lambda_{j}^{*} a^{\dagger} e^{i \delta t}\right)\left|1_{j}\right\rangle\left\langle 1_{j}\right|\right]
\end{aligned}
$$

where $\delta=\Delta_{2}-\Delta_{1}, \lambda_{j}=\Omega_{j}^{*} g_{j}\left(1 / \Delta_{1}+1 / \Delta_{2}\right) / 2$. The first and second terms in Eq. (2) are the Stark shifts for the level $\left|1_{j}\right\rangle$ that are induced by the cavity mode and the classical pulse, respectively. The last term is the coupling between the cavity mode and the classical field assisted by the atoms. In the case of $\delta \gg \frac{\left|\Omega_{j}\right|^{2}}{\Delta_{2}}, \frac{\left|g_{j}\right|^{2}}{\Delta_{1}}$, $\left|\lambda_{j}\right|$, the cavity photon is only virtually excited and any two atoms interfere with each other [35]. For the sake of convenience, we will assume $\Omega_{j}=\Omega, g_{j}=g$, and $\Omega$ and $g$ are real so that $\lambda_{j}=\Omega g\left(1 / \Delta_{1}+1 / \Delta_{2}\right) / 2=\lambda$. The effective Hamiltonian is then given by

$$
\begin{aligned}
H_{e f f} & =\sum_{j=1}^{N}\left(-\frac{g^{2}}{\Delta_{1}} a^{\dagger} a-\frac{\Omega^{2}}{\Delta_{2}}+\frac{\lambda^{2}}{\delta}\right)\left|1_{j}\right\rangle\left\langle 1_{j}\right| \\
& +\lambda^{\prime} \sum_{j, k=1, j \neq k, j<k}^{N}\left|1_{j}\right\rangle\left\langle 1_{j}|| 1_{k}\right\rangle\left\langle 1_{k}\right|,
\end{aligned}
$$

where $\lambda^{\prime}=\frac{2 \lambda^{2}}{\delta}$. The last term in Eq. (3) describes the coupling between atoms $\mathrm{j}$ and $\mathrm{k}$ mediated by the cavity mode and the classical pulse. If the cavity field is initially in the vacuum state, the effective Hamiltonian reduces to

$$
\begin{aligned}
H_{e f f} & =\sum_{j=1}^{N}\left(-\frac{\Omega^{2}}{\Delta_{2}}+\frac{\lambda_{j}^{2}}{\delta}\right)\left|1_{j}\right\rangle\left\langle 1_{j}\right| \\
& +\lambda^{\prime} \sum_{j, k=1, j \neq k, j<k}^{N}\left|1_{j}\right\rangle\left\langle 1_{j}|| 1_{k}\right\rangle\left\langle 1_{k}\right| .
\end{aligned}
$$

Suppose that the two low levels $|0\rangle$ and $|1\rangle$ of each atom represent two logical states of a qubit. The time evolutions of four logical states for two qubits, under the Hamiltonian (4), are given by

$$
\begin{aligned}
\left|0_{j}\right\rangle\left|0_{k}\right\rangle & \rightarrow\left|0_{j}\right\rangle\left|0_{k}\right\rangle, \\
\left|0_{j}\right\rangle\left|1_{k}\right\rangle & \rightarrow e^{-i \xi_{I}}\left|0_{j}\right\rangle\left|1_{k}\right\rangle, \\
\left|1_{j}\right\rangle\left|0_{k}\right\rangle & \rightarrow e^{-i \xi_{I}}\left|1_{j}\right\rangle\left|0_{k}\right\rangle, \\
\left|1_{j}\right\rangle\left|1_{k}\right\rangle & \rightarrow e^{-i\left(2 \xi_{I}+\phi\right)}\left|1_{j}\right\rangle\left|1_{k}\right\rangle,
\end{aligned}
$$

where $\xi_{I}=\left(-\frac{\Omega^{2}}{\Delta_{2}}+\frac{\lambda^{2}}{\delta}\right) t$ and $\phi=\lambda^{\prime} t$. After the performance of the one-qubit operation $\left|1_{j(k)}\right\rangle \rightarrow e^{i \xi_{I}}\left|1_{j(k)}\right\rangle$, there is

$$
\begin{aligned}
\left|0_{j}\right\rangle\left|0_{k}\right\rangle & \rightarrow\left|0_{j}\right\rangle\left|0_{k}\right\rangle, \\
\left|0_{j}\right\rangle\left|1_{k}\right\rangle & \rightarrow\left|0_{j}\right\rangle\left|1_{k}\right\rangle, \\
\left|1_{j}\right\rangle\left|0_{k}\right\rangle & \rightarrow\left|1_{j}\right\rangle\left|0_{k}\right\rangle \\
\left|1_{j}\right\rangle\left|1_{k}\right\rangle & \rightarrow e^{-i \phi}\left|1_{j}\right\rangle\left|1_{k}\right\rangle .
\end{aligned}
$$

Thus a conditional phase shift $\phi=\lambda^{\prime} t$ is produced if and only if two atoms both are in the state $|1\rangle$. The $\mathrm{n}$ times similar operations can accumulate a phase $\phi^{\prime}=$ $\sum_{i}^{n} \int_{t_{i}}^{t_{(i+1)}} \lambda_{i}^{\prime} d t$ varying between 0 and $2 \pi$, which is controllable via the total effective interaction time $t$ ( $t_{i}$ denotes the beginning time of the ith operation). Ref. [36] shows that any conditional quantum phase gate is universal, since all quantum computations can be realized by combining it and rotations of individual qubits. For example, with the choice of $t=\frac{\pi}{\lambda^{\prime}}$, a two-qubit controlled-Z gate is obtained, which is a familiar two-qubit universal logic 
gate [37]. We note that Yi et al. have proposed a novel scheme for conditional quantum phase gate between two 3 -state atoms via simultaneously driven by a single-mode cavity field and a very weak classical field [34]. However, there are the following differences between our protocol and that in Ref. [34]. (a) Our scheme does not need the wave front delay of the classical field between the two atoms corresponds to an odd number of $\pi$, which require that two atoms are separated from each other by at least half optical wavelength of the classical field. (b) Our method can be extended to construct multiple-qubit entangling gates, which are used as basic tools for effective generation of graph-state entanglement in next section. But the scheme in Ref. [34] is difficult to simultaneously operate a controlled phase gate between two arbitrary atoms coupled to the cavity, due to one can't make the wave front delay of the classical field between arbitrary two of several atoms correspond to an odd number of $\pi$. (c) Unlike the scheme in Ref. [34, our scheme doesn't require weak classical field $(\Omega<g)$ for the large detuning.

\section{EFFECTIVE GENERATION OF GRAPH-STATE ENTANGLEMENT}

We first give a brief review of the definition and properties of graph states [18, 19]. An n-qubit graph state is defined as the coeigenstate of $\mathrm{n}$ independent stabilizer operators $S_{i}=X_{i} \Pi_{j} Z_{j}$, where i denotes qubit i (each qubit is associated with a vertex of the graph), j runs over all the neighbors of the qubit $i$, and $X_{i}, Z_{i}$ are simply the Pauli operators $\sigma_{x}$ and $\sigma_{z}$ for qubit $i$. In a graph state, the qubits $i$ and $j$ are called neighbor if they are connected with an edge. The graph state reduces to a cluster state if the corresponding graph is a periodic lattice [26]. Each graph can be represented by a diagram in a plane, where each vertex is represented by a point and each edge by an arc joining two not necessarily distinct vertices. In this pictorial representation many concepts related to graphs can be visualized in a transparent manner. A graph $|G\rangle$ is local unitary (LU) equivalent representation of another graph $\left|G^{\prime}\right\rangle$, if the graph $|G\rangle$ can be transformed into $\left|G^{\prime}\right\rangle$ only by local unitary operation. It has been proved that, by local complementation of a graph $|G\rangle$ at one vertex and leaving the rest of the graph $|G\rangle$ unchanged, a new graph $\left|G^{\prime}\right\rangle$ obtained is LUequivalent representation of the graph $|G\rangle$. This is called LU rule [19] (or LC rule [18]).

The graph state $|G\rangle$ can be obtained by applying a sequence of commuting unitary two-qubit controlled$\mathrm{Z}$ gate $U_{z}^{(a, b)}$ to the empty graph $|+\rangle^{\otimes n}:|G\rangle=$ $\Pi_{(a, b) \in E} U_{z}^{(a, b)}|+\rangle^{\otimes n}$, where $E$ denotes the set of edges in the graph $|G\rangle$, and $|+\rangle=(|0\rangle+|1\rangle) / \sqrt{2}$. The unitary two-qubit operation $U_{z}^{(a, b)}$ on the vertices $a, b$ adds or removes the edge $\{a, b\}$. In the following, with a combination of the LU rule and the multiple-qubit entan- gling gates, we show an efficient scheme for preparation of graph-state entanglement.

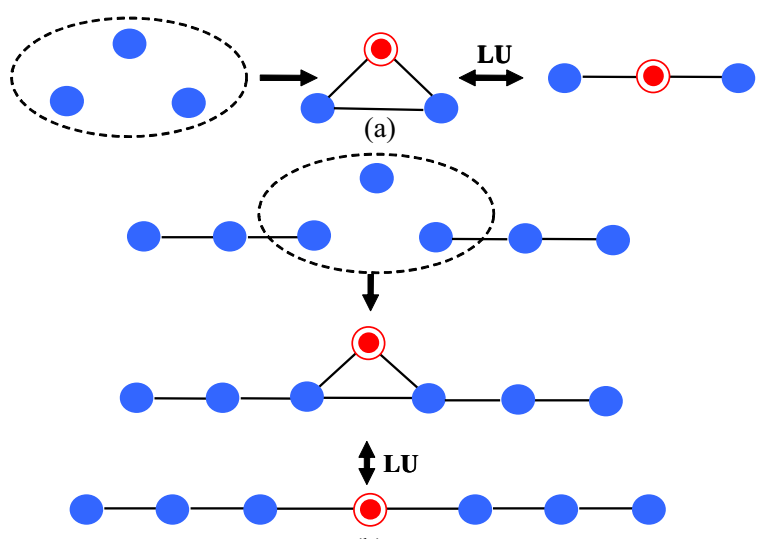

(b)

FIG. 2: (Color online) (a) The representation of the threequbit entangling gate. (b) Illustration of fusing two threequbit linear cluster states into a larger one by a three-qubit entangling gate. The ringed point denotes the vertex, to which the LU rule is applied. The notation LU denotes that two graph states can be transformed into each other only by local unitary operation.

Three-qubit entangling gate.- As shown in Fig. 2(a), three atoms 1,2 , and 3 with the initial state $\left|\Phi_{3}\right\rangle=$ $\frac{1}{\sqrt{2^{3}}} \Pi_{j=1}^{3}\left(|0\rangle_{j}+|1\rangle_{j}\right)$ trapped in a cavity are simultaneously driven by the cavity mode and an appropriate classical pulse (Fig. 1). The state evolution of the three-atom system is governed by

$$
H_{e f f}=\lambda^{\prime} \sum_{j \neq k, j<k}\left|1_{j}\right\rangle\left\langle 1_{j}|| 1_{k}\right\rangle\left\langle 1_{k}\right|,
$$

where $j, k=1,2,3, \lambda^{\prime}=\frac{2 \lambda^{2}}{\delta}$, and the self-energy terms have been assumed to be corrected by the added classical pulses [38]. After the effective interaction time $t=\frac{\pi}{\lambda^{\prime}}$, a three-qubit entangling gate is operated on the three-atom system and a graph state

$$
\left|\Phi_{3}^{\prime}\right\rangle=U_{z}^{(1,2)} U_{z}^{(2,3)} U_{z}^{(1,3)}\left|\Phi_{3}\right\rangle
$$

is obtained. The graph state is LU-equivalent representation of the three-qubit linear cluster. Fig. 2(b) shows that two three-qubit linear clusters are fused into a sevenqubit graph state by a three-qubit entangling gate, which is LU-equivalent representation of a seven-qubit linear cluster. With sequentially applying the method in Fig. 2, we can get $n$-qubit ( $\mathrm{n}$ is odd) linear cluster state through $\frac{n-1}{2}$ times successful three-qubit entangling gates.

Four-qubit entangling gate.- In similar way, as sketched in Fig. 3(a), four atoms 1, 2, 3, and 4 with the initial state $\left|\Phi_{4}\right\rangle=\frac{1}{\sqrt{2^{4}}} \Pi_{j=1}^{4}\left(|0\rangle_{j}+|1\rangle_{j}\right)$ simultaneously interact with a cavity mode and an appropriate 


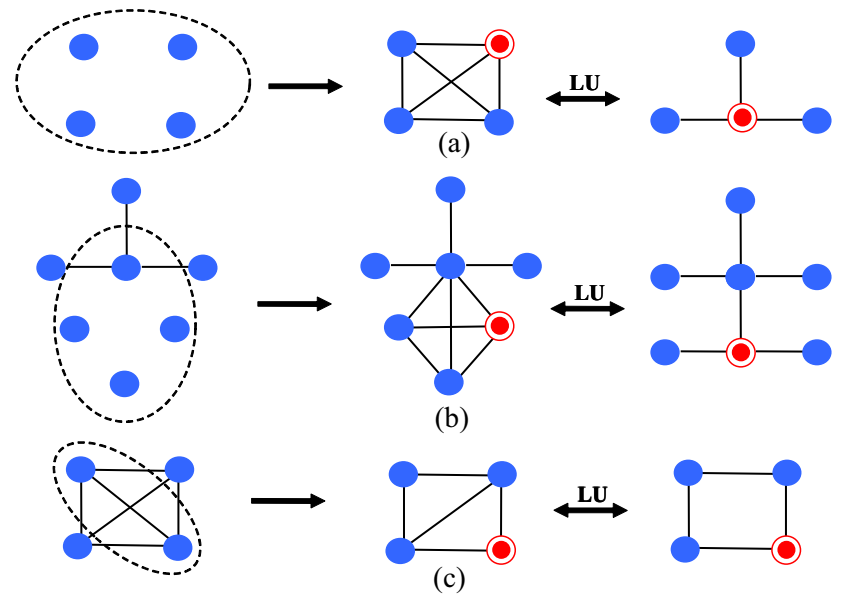

FIG. 3: (Color online) (a) The representation of the fourqubit entangling gate. (b) Illustration of using the four-qubit entangling gates to generate a H-sharp graph state. (c) Illustration of constructing a box graph by performing an added controlled-Z gate on two diagonal vertexes of the graph in (a). Other notations are the same as Fig. 2.

classical pulse (Fig. 1). After the effective interaction time $t=\frac{\pi}{\lambda^{\prime}}$, the state evolution of four-atom system is

$$
\left|\Phi_{4}^{\prime}\right\rangle=U_{z}^{(1,2)} U_{z}^{(1,3)} U_{z}^{(1,4)} U_{z}^{(2,3)} U_{z}^{(2,4)} U_{z}^{(3,4)}\left|\Phi_{4}\right\rangle,
$$

which is a four-qubit graph state. The graph state is LU-equivalent representation of a four-qubit star graph, which is equivalent to four-particle GHZ state [18, 19]. Fig. 3(b) shows that with two four-qubit entangling gates one can obtain a seven-qubit graph state, which is LUequivalent representation of an H-sharp graph. Fig. 3(c) shows that after performing an controlled- $Z$ gate on two diagonal vertexes of the graph in Fig. 3(a) 39], we can obtain another class graph state, which is LU-equivalent representation of a box graph.

Five or more-qubit entangling gate.-Five or more atoms simultaneously interact with a cavity mode and an appropriate classical pulse. After the effective interaction time $t=\frac{\pi}{\lambda^{\prime}}$, we obtain a five or more-qubit entangling gate. As sketched in Fig. 4(a), after applying a five-qubit entangling gate, one can get a five-qubit graph sate, which is LU-equivalent representation of a five-qubit star graph state being equivalent to a five-particle GHZ state [18, 19]. Fig. 4(b) shows that a eight-qubit 2D graph state can be obtianed by applying two five-qubit entangling gates. Fig. 4(c) shows how to effectively generate $2 \mathrm{D}$ square lattice cluster states by using the fivequbit entangling gates, which can be used as the essential resource for realization of universal quantum computation [26]. In Fig. 5, we illustrate a method for effective preparation of three-dimensional (3D) cluster state by sequentially using the seven-qubit entangling gates. In Ref. [40], Raussendorf et al. have shown that 3D cluster state is a fault-tolerant fabric of one-way quantum computer.

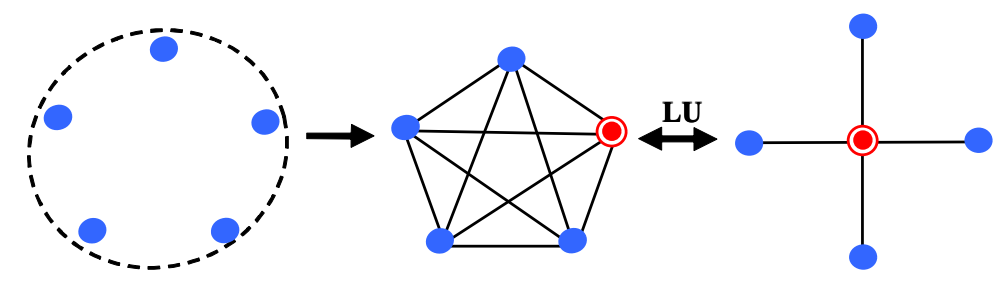

(a)

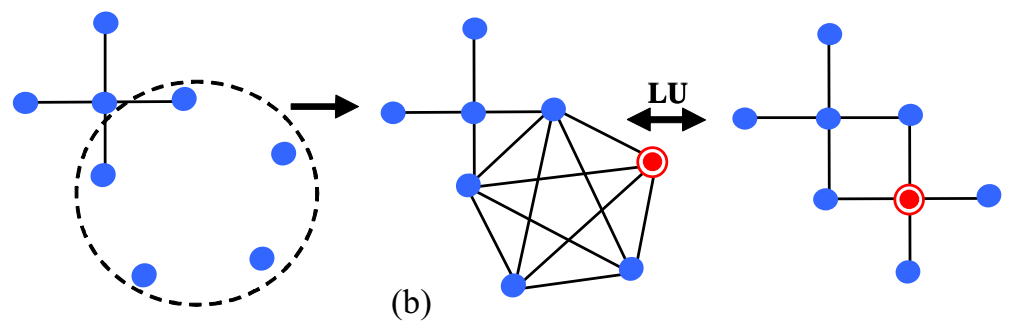

FIG. 4: (Color online) (a) The representation of the fivequbit entangling gate. (b) and (c) Illustration of using the five-qubit entangling gates to generate square lattice cluster states. Other notations are the same as Fig. 2.

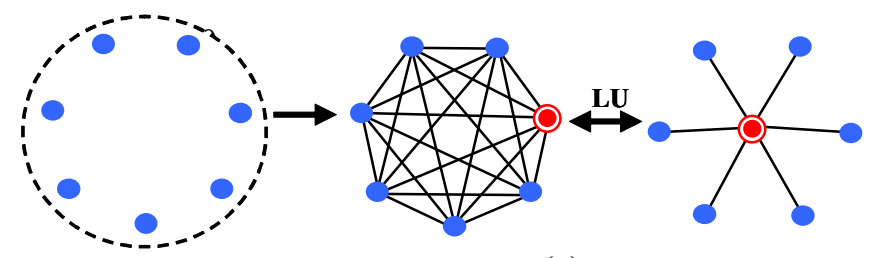

(a)

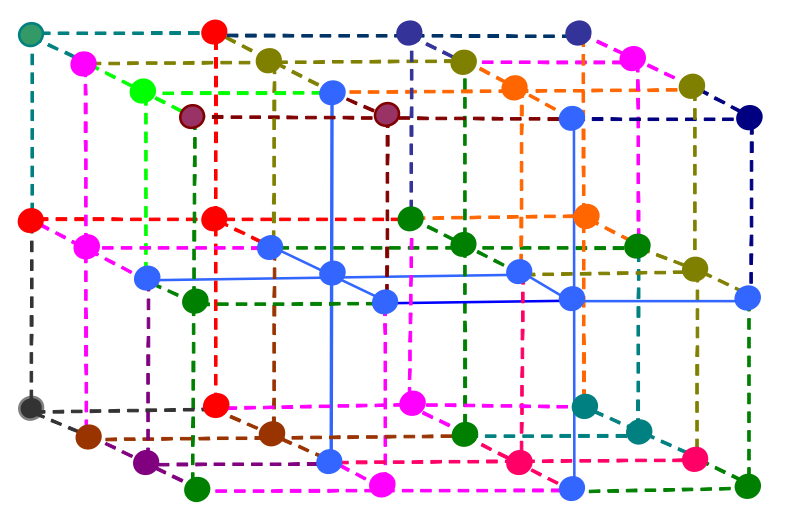

(c)

FIG. 5: (Color online) (a) The representation of the sevenqubit entangling gate. (b) and (c) Illustration of using the seven-qubit entangling gates to generate $3 \mathrm{D}$ cluster states. Other notations are the same as Fig. 2.

Here we only display a sample of generating some typical graph states. We believe that it is still effective to generate a more complex graph state by combining the LU rule, the multiple-qubit entangling gates, and any two-qubit controlled-Z gate. 


\section{DISCUSSION AND CONCLUSION}

The required atomic level configuration and transitions can be achieved in neutral atoms trapped in an optical cavity, solid-state qubits - often called artificial atoms trapped in a microwave cavity, or the ions trapped in the potential trap. It should be noted that one needs to reach the strong-coupling limit and the Lamb-Dicke regime in order to perform the gate successfully. In optical cavity QED system, strong-coupling limit and localization to the ground state of motion for an atom trapped in an optical cavity are still a challenging pursuit [41]. Conversely, for the solid-state system or iontrap system, strong-coupling and Lamb-Dicke limit can be easier to achieve [42]. In the past few years, much attention has been paid to superconducting devices such as Cooper pair boxes, Josephson junctions, and superconducting quantum interference devices (SQUIDs), since these solid-state artificial atoms are relatively easy to scale up and have been demonstrated to have relatively long decoherence time [43, 44]. Recently, for SQUID system, many methods for realizing a single-qubit arbitrary rotation gate and a two-qubit controlled-NOT (or controlled-Z gate) have been presented [45, 46]. Any particular SQUID qubit operation can be realized by adjusting level structure of individual qubit [45]. In the following we first discuss that it is possible to experimentally demonstrate our scheme with the SQUIDs in a microwave cavity.

Typically, for SQUIDs interacting with a high-Q microwave cavity, the coupling constant is $g \sim 1.8 \times 10^{8} \mathrm{~Hz}$, the photon lifetime (the energy relaxation time of excited state $|e\rangle)$ is $t_{c(r)} \sim 7.6 \times 10^{-7} s$ [45]. With the choices of $\Omega=1.05 \mathrm{~g}, \Delta_{1}=20 \mathrm{~g}, \Delta_{2}=21 \mathrm{~g}$, we have $\delta=\Delta_{2}-\Delta_{1}=g \sim 20 \frac{g^{2}}{\Delta_{1}}, 19.05 \frac{\Omega^{2}}{\Delta_{2}}, 19.5 \lambda$, which satisfy the conditions $\Delta_{1} \gg g, \Delta_{2} \gg \Omega$, and $\delta \gg \frac{g^{2}}{\Delta_{1}}, \frac{\Omega^{2}}{\Delta_{2}}, \lambda$. Our calculations show that (i) the occupation probability of the excited state $|e\rangle$ is $P_{r} \sim 0.01\left(\simeq \frac{\Omega^{2}}{\delta^{2}}\right)$, thus the effective energy relaxation time is $t_{r}^{\prime} \sim P_{r}^{-1} t_{r} \sim 76 \mu \mathrm{s}$; (ii) the occupation probability of the photon is $P_{c} \sim 0.01\left(\simeq \frac{\lambda^{2}}{\delta^{2}}\right)$, thus the effective photon lifetime is $t_{c}^{\prime} \sim P_{c}^{-1} t_{c} \sim 76 \mu \mathrm{s}$; (iii) the required effective interaction time for a two-qubit controlled- $\mathrm{Z}$ gate or a multiple-qubit entangling gate is $t \sim \frac{\pi}{\lambda^{\prime}} \sim 3 \mu s$. Therefore, it is possible to perform several two-qubit controlled-Z gates or multiple-qubit entangling gates within the decoherence time $t_{r}^{\prime} \sim t_{c}^{\prime} \sim 76 \mu \mathrm{s}$.

In the ion-trap system, several ions are assumed to be confined in a linear trap [5, 6] and have been cooled to the ground state. The ions are simultaneously excited by two laser fields (for operating a controlled phase gate between two or several arbitrary ions, one needs single laser pulses to address two or several arbitrary ions individually, which have been demonstrated in the experiment [47]). One is (off-resonantly) tuned to the first lower vibrational side and the other is insensitive to the motion with appropriate frequency [34], as shown in Fig. 1. In the Lamb-Dicke limit the Hamiltonian of the system is then again described by Eq. (1), except now $a$ is the annihilation operator of the collective vibrational mode instead of the cavity mode. Considering experimental parameters of ion experiments at Ref. [10], we can achieve an effective coupling rate $\lambda^{\prime} \sim 10^{4} \mathrm{~Hz}$. So the required effective interaction time for a two-qubit controlled- $Z$ gate or a multiple-qubit entangling gate is $t \sim 0.1 \mathrm{~ms}$, shorter than the typical motional decoherence time $t_{d} \sim 10 \mathrm{~ms}$.

In summary, we have proposed a scheme to realize a tunable quantum phase gate with three-level atoms or artificial atoms or ions simultaneously interacting with a quantum data bus such as a single mode field in cavity QED system, or a collective vibrational state of trapped ions. The distinct advantages of our scheme are that there is no cavity-photon population involved and the atoms are always in their low levels. In addition, our method can be extended to construct multiplequbit graph-state entanglement. The method opens up a prospect to generate large-scale graph state in cavity QED system and ion trap system.

Acknowledgments: This work was funded by $\mathrm{Na}$ tional Natural Science Foundation of China (Grant No. 10574022), the Natural Science Foundation of Fujian Province of China (Grant No. 2007J0002), and the Foundation for Universities in Fujian Province (Grant No. 2007F5041).
[1] P. Shor, in Proceedings of the 35th Annual Symposium on the Foundation of Computer Science (IEEE Computer Society Press, Los Alamitos, CA, 1994), pp. 124-134; L. K. Grover, Phys. Rev. Lett. 79, 325 (1997).

[2] C. H. Bennett and S. J. Wiesner, Phys. Rev. Lett. 69, 2881 (1992); C. H. Bennett et al., Phys. Rev. Lett. 70, 1895 (1993).

[3] T. Sleator and H. Weinfurter, Phys. Rev. Lett. 74, 4087 (1995).

[4] S. B. Zheng and G C Guo, Phys. Rev. Lett. 85, 2392 (2000).

[5] J. I. Cirac and P. Zoller, Phys. Rev. Lett. 74, 4091 (1995).

[6] Anders SØrensen and Klaus MØlmer, Phys. Rev. Lett.
82, 1971 (1999).

[7] G. Nogues, A. Rauschenbeutel, S. Osnaghi, M. Brune, J. M. Raimond, and S. Haroche, Nature (London) 400, 239 (1999).

[8] J. McKeever, A. Boca, A. D. Boozer, R. Miller, J. R. Buck, A. Kuzmich, and H. J. Kimble, Science 303, 1992 (2004); A. D. Boozer, A. Boca, R. Miller, T. E. Northup, and H. J. Kimble, Phys. Rev. Lett. 97, 083602 (2006).

[9] P. Maunz, T. Puppe, I. Schuster, N. Syassen, P. W. H. Pinkse and G. Rempe, Nature (London) 428, 50 (2004); P. Maunz, T. Puppe, I. Schuster, N. Syassen, P. W. H. Pinkse, and G. Rempe, Phys. Rev. Lett. 94, 033002 (2005). 
[10] C. A. Sackett, D. Kielpinski, B. E. King, C. Langer, V. Meyer, C. J. Myatt, M. Rowe, Q. A. Turchette, W. M. Itano, D. J. Wineland, and C. Monroe, Nature (London) 404, 256 (2000).

[11] D. Leibfried, R. Blatt, C. Monroe, and D. Wineland, Rev. Mod. Phys. 75, 281 (2003).

[12] E. Solano et al., Phys. Rev. A 64, 024304 (2001); M. S. Zubairy et al., Phys. Rev. A 68, 033820 (2003); S. RebiĆ et al., Phys. Rev. A 70, 032317 (2004); X. M. Lin et al., Phys. Rev. A 73, 012323 (2006); X. B. Zou et al., Phys. Rev. A 75, 064301 (2007); J. F. Poyatos et al., Phys. Rev. Lett. 81, 1322 (1998); C. Di Fidio et al., Phys. Rev. A 65, 033825 (2002); P. Staanum et al., Phys. Rev. A 66, 040302 (2002); F. Mintert et al., Phys. Rev. Lett. 87, 257904 (2001); F. L. Semião et al., Phys. Rev. A 72, 064305 (2005); X. M. Lin et al. Phys. Rev. A 74, 052339 (2006); L. Aolita et al., Phys. Rev. A 75, 052337 (2007).

[13] E. Hagley et al., Phys. Rev. Lett. 79, 1 (1997); S. Osnaghi et al., ibid. 87, 037902 (2001).

[14] A. Rauschenbeutel et al., Science 288, 2024 (2000).

[15] M. Brune et al., Phys. Rev. Lett. 77, 4887 (1996).

[16] S. Brattke, B. T. H. Varcoe, and H. Walther, Phys. Rev. Lett. 86, 3534 (2001); P. Bertet et al., ibid. 88, 143601 (2002).

[17] H Häffner et al., Nature 438, 643 (2005).

[18] M. Hein, W. Dür, J. Eisert, R. Raussendorf, M. Van den Nest, H.-J. Briegel, e-print quant-ph/0602096

[19] M. Hein, J. Eisert, and H. J. Briegel, Phys. Rev. A 69, 062311 (2004).

[20] V. Scarani, A. Acín, E. Schenck, and M. Aspelmeyer, Phys. Rev. A 71, 042325 (2005).

[21] Otfried GÜhne, Géza Tóth, Philipp Hyllus, and Hans J. Briegel, Phys. Rev. Lett. 95, 120405 (2005).

[22] M. Hillery, V. Bužek., and A. Berthiaume, Phys. Rev. A 59, 1829 (1999); D. Gottesman, Phys. Rev. A 61, 042311 (2000).

[23] D. Schlingemann and R. F. Werner, Phys. Rev. A 65, 012308 (2002).

[24] D. Gottesman, Ph.D. thesis, CalTech, Pasadena, CA, (1997).

[25] W. Dür and H.-J. Briegel, Phys. Rev. Lett. 92, 180403 (2004).

[26] R. Raussendorf and H. J. Briegel, Phys. Rev. Lett. 86, 5188 (2001).

[27] M. Varnava, D. E. Browne, and T. Rudolph, Phys. Rev. Lett. 97, 120501 (2006).

[28] T. P. Bodiya and L.-M. Duan, Phys. Rev. Lett. 97, 143601 (2006).

[29] A. Kay and J. K. Pachos, Phys. Rev. A 73, 022310 (2006).

[30] S. R. Clark, C. Moura-Alves, and D. Jaksch, New J.
Phys. 7, 124 (2005).

[31] C. Y. Lu et al., nature physics 3, 91 (2007).

[32] A. Rauschenbeutel, G. Nogues, S. Osnaghi, P. Bertet, M. Brune, J. M. Raimond, and S. Haroche, Phys. Rev. Lett. 83, 5166 (1999).

[33] S. B. Zheng and G. C. Guo, Phys. Rev. A 73, 052328 (2006).

[34] X. X. Yi, X. H. Su, and L. You, Phys. Rev. Lett. 90, 097902 (2003).

[35] K. MØlmer and A. SØrensen, Phys. Rev. Lett. 82, 1835 (1999); G. W. Lin et al., Phys. Rev. A 76, 014308 (2007); A. Imamoglu, D. D. Awschalom, G. Burkard, D. P. DiVincenzo, D. Loss, M. Sherwin, and A. Small, Phys. Rev. Lett. 83, 4204 (1999).

[36] M. J. Bremner, C. M. Dawson, J. L. Dodd, A. Gilchrist, A. W. Harrow, D. Mortimer, M. A. Nielsen, and T. J. Osborne, Phys. Rev. Lett. 89, 247902 (2002).

[37] A. Barenco, C. H. Bennett, R. Cleve, D. P. DiVincenzo, N. Margolus, P. Shor, T. Sleator, J. A. Smolin, and H.Weinfurter, Phys. Rev. A 52, 3457 (1995).

[38] A. Rauschenbeutel, G. Nogues, S. Osnaghi, P. Bertet, M. Brune, J. M. Raimond, and S. Haroche, Science 288, 2024 (2000).

[39] For operating a controlled phase gate between two arbitrary atoms trapped in the cavity, one need the atoms are separated by at least one optical wavelength so that single laser pulses can address each atom individually, which is difficult to achieve with the current experimental technology.

[40] R. Raussendorf, J. Harrington and K. Goyal, e-print quant-ph/0510135.

[41] Tatjana Wilk et al., Science 317, 488 (2007).

[42] A. Wallraff, D. I. Schuster, A. Blais, L. Frunzio, R. S. Huang, J. Majer, S. Kumar, S. M. Girvin, and R. J. Schoelkopf, Nature 431, 162 (2004).

[43] D. Vion, A. Aassime, A. Cottet, P. Joyez, H. Pothier, C. Urbina, D. Esteve, and M. H. Devoret, Science 296, 886 (2002).

[44] Y. Yu, S. Han, X. Chu, Shih-I Chu, and Z. Wang, Science 296, 889 (2002).

[45] C. P. Yang and Shih-I Chu, Phys. Rev. A 67, 042311 (2003); C. P. Yang, Shih-I Chu, and Siyuan Han, Phys. Rev. Lett. 92, 117902 (2004).

[46] C. P. Yang and Siyuan Han, Phys. Rev. A 73, 032317 (2006); K. H. Song et al., Phys. Rev. A 75, 032347 (2007); C. P. Yang et al., Phys. Rev. A 70, 044303 (2004).

[47] F. Schmidt-Kaler et al., Nature (London) 422, 408 (2003). 